\title{
„Wir müssen jetzt politischen Druck erzeugen"
}

\begin{abstract}
Seit sechs Jahrzehnten ist die Approbationsordnung für Zahnärzte weitgehend unverändert. Das stößt nicht nur bei den Studenten auf deutliche Kritik. Selbst die Politik erkannte schon vor einigen Jahren die Notwendigkeit einer Neufassung an. Doch die aktuelle Bundesregierung blockiert derartige Bestrebungen. Zunächst soll ein "Masterplan Medizin 2020" entwickelt werden. Die Zahnärzteschaft sieht darin eine Verzögerungstaktik und schließt Konsequenzen nicht aus.
\end{abstract}

Der Konsens über die Notwendigkeit einer neuen Approbationsordnung für Zahnärzte ist schon alt. Bereits der Koalitionsvertrag der Bundesregierung in der 17. Legislaturperiode von 2009 bis 2013 sah vor, die Approbationsordnung zu novellieren. Auch die Kultusministerkonferenz hatte dies anerkannt. Doch die derzeitige Bundesregierung hat offenbar vorerst andere Pläne und will vorrangig den „Masterplan Medizin 2020“ realisieren. Den hat die Große Koalition in ihrem Koalitionsvertrag angekündigt und arbeitet derzeit zusammen mit den Bundesländern an einer Reform des Medizinstudiums. Ziele sind unter anderem Maßnahmen für eine zielgerichtete Auswahl der Studienplatzbewerber, zur Förderung der Praxisnähe und zur Stärkung der Allgemeinmedizin im Studium. Eine Bund-Länder-Arbeitsgruppe soll bis Mitte 2016 ein entsprechendes Reformkonzept vorlegen.

Und wann steht dann die die Approbationsordnung für die Zahnärzte auf dem Programm? Die Bundesversammlung der Bundeszahnärztekammer (BZ̈̈K) hat die Bundesregierung im Oktober 2015 aufgefordert, die Neufassung der Approbationsordnung samt deren Aussagen zur Ausbildungskapazität noch in dieser Legislaturperiode umzusetzen (siehe hierzu auch Seite 25). Das Bundesgesundheitsministerium (BMG) hielt sich jedoch auf DFZ-Anfrage bedeckt, was den genauen Zeitplan angeht. In einem Statement des BMG heißt es: „Um zeitnah eine Anpassung des Studiums der Zahnmedizin an die fachliche Weiterentwicklung der Zahnmedizin zu ermöglichen, wurde zum 1. Januar 2016 durch eine Änderung des Gesetzes über die Ausübung der Zahnheilkunde die rechtliche Grundlage zur Durchführung von Modellstudiengängen im Studiengang Zahnmedizin geschaffen. Am Ziel einer Gesamtreform des Studiums der Zahnmedizin wird uneingeschränkt festgehalten."

\section{Modellstudiengänge - ein fauler Kompromiss}

Nicht nur die BZ̈̈K, sondern auch der Bundesvorsitzende des Freien Verbands Deutscher Zahnärzte (FVDZ), Harald Schrader, hält das Vorgehen der Bundesregierung für nicht hinnehmbar. „Das ist eine inakzeptable Verzögerungstaktik“, meint Schrader. „Denn wenn man die Approbationsordnung verabschiedet, dann würde man sich die Akademisierung der Fachberufe verbauen. “Ziel der Modellstudiengänge sei offenbar, das Staatsexamen aufzuweichen, ist der FVDZ-Vorsitzende über-

zeugt. Mit der Öffnung von Modellstudiengängen werde der Weg für uneinheitliche Ausbildungen frei gemacht. „Und deshalb ist das ein fauler Kompromiss“, kritisiert Schrader.

Für Jan-Philipp Schmidt vom Bundesverband der zahnmedizinischen Alumni in Deutschland (BdZA) hat die Verzögerung einen ganz entscheidenden Grund: „Der Mehraufwand eines modernen Studiums erfordert entsprechendes Lehrpersonal. Und da würden auf die Unis deutlich mehr Ausgaben zukommen. Deshalb wollen sie die Approbationsordnung nicht angehen“, sagt der BdZA-Vorsitzende.

\section{Bundesweiter Streik geplant}

Doch die Zahnärzteschaft will einen weiteren Stillstand nicht so einfach hinnehmen. Die Forderungen nach einer Novellierung der 60 Jahre alten Approbationsordnung werden schärfer. Zahnmedizinstudenten und Standespolitiker sind sich einig, dass der Blockade des BMG notfalls auch mit Streiks begegnet werden müsse. „Wir planen im Mai einen dreitägigen bundesweiten Streik an den Unis“, kündigt der Vorsitzende des Bundesverbands der Zahnmedizinstudenten in Deutschland (BdZM), Kai Becker, an. Und so, wie es aussehe, wollten die Fachschaften auch relativ geschlossen mitmachen. „Wir müssen jetzt politischen Druck erzeugen “, ist Becker überzeugt und bekommt breite Unterstützung. Sowohl die BZÄK als auch der FVDZ und der BdZA - die Vertretung der Uni-Absolventen stärken dem zahnmedizinischen Nachwuchs im Schulterschluss den Rücken. „Wenn keine neue Approbationsordnung kommt, dann müssen die Studenten eben mal streiken “, sagt BdZA-Chef Schmidt. „Denn wenn man nicht streikt, dann ändert sich auch nichts." 